Article

\title{
Methods for MADM with Picture Fuzzy Muirhead Mean Operators and Their Application for Evaluating the Financial Investment Risk
}

\author{
Rui Wang, Jie Wang *, Hui Gao and Guiwu Wei * \\ School of Business, Sichuan Normal University, Chengdu 610101, China; wangrui_sicnu@163.com (R.W.); \\ gaohuisxy@sicnu.edu.cn (H.G.) \\ * Correspondence: JW970326@163.com (J.W.); weiguiwu1973@sicnu.edu.cn (G.W.)
}

Received: 4 December 2018; Accepted: 17 December 2018; Published: 21 December 2018

\begin{abstract}
In this article, we study multiple attribute decision-making (MADM) problems with picture fuzzy numbers (PFNs) information. Afterwards, we adopt a Muirhead mean (MM) operator, a weighted MM (WMM) operator, a dual MM (DMM) operator, and a weighted DMM (WDMM) operator to define some picture fuzzy aggregation operators, including the picture fuzzy MM (PFMM) operator, the picture fuzzy WMM (PFWMM) operator, the picture fuzzy DMM (PFDMM) operator, and the picture fuzzy WDMM (PFWDMM) operator. Of course, the precious merits of these defined operators are investigated. Moreover, we have adopted the PFWMM and PFWDMM operators to build a decision-making model to handle picture fuzzy MADM problems. In the end, we take a concrete instance of appraising a financial investment risk to demonstrate our defined model and to verify its accuracy and scientific merit.
\end{abstract}

Keywords: MADM; picture fuzzy set (PFS); PFMM operator; PFWMM operator; PFDMM operator; PFWDMM operator; financial investment risk

\section{Introduction}

Atanassov [1] defined intuitionistic fuzzy sets (IFSs), which are an extension of fuzzy sets (FSs) [2]. Atanassov and Gargov [3] and Atanassov [4] presented the definition of interval-valued IFSs (IVIFS); since then, FSs, IFSs, and IVIFSs have attracted more and more scholars' and researchers' attention [5-16]. Recently, Cuong and Kreinovich [17] developed the picture fuzzy set (PFS), and studied some basic operations, rules, and properties of PFS. Singh [18] explored the correlation coefficients of PFS. Son etc. $[19,20]$ presented several novel fuzzy clustering algorithms based on PFSs. Thong and Son [21] defined a novel hybrid model between picture fuzzy clustering and intuitionistic fuzzy recommender systems for medical diagnosis and application to health care support systems. Wei [22] proposed the picture fuzzy cross-entropy method to deal with multiple attribute decision-making (MADM) problems. Thong and Son [23] gave Automatic Picture Fuzzy Clustering (AFC-PFS) for determining the most suitable number of clusters for FC-PFS. Wei [24] assigned some cosine similarity measures of PFSs for strategic decision-making on the basis of traditional similarity measures [25-29]. Wei [30] also defined some similarity measures for PFSs. Wei [31] defined some aggregation operators for MADM problems with respect to PFSs based on traditional aggregation operators [14,32-37]. Wei [38] proposed some picture fuzzy Hamacher aggregation operators with traditional Hamacher operations [39-42]. Zhang et al. [43] provided some relative projection models for PFSs. Wei [44] proposed the TODIM model for picture fuzzy MADM problems. Wang et al. [45] formulated a hybrid fuzzy MADM framework with PFSs. Wei et al. [46] designed PFN projection models to handle MADM problems. Wei [47] defined some picture 2-tuple linguistic Bonferroni mean 
operators in MADM. Wei et al. [48] proposed some picture 2-tuple linguistic operators. Wei [49] defined some picture uncertain linguistic Bonferroni mean operators. Wang and Li [50] combined PFSs and the hesitant fuzzy set [51-56] to propose the picture hesitant fuzzy set (PHFS) theory.

The Muirhead mean (MM) [57] is a useful decision-making tool that can identify the inter-relationships among any number of information fusions, and some existing operators, such as arithmetic and geometric operators (not considering the inter-relationships). Both the Bonferroni mean (BM) operator [58-63] and the Maclaurin symmetric mean (MSM) operator [8,64] are special issues in the MM operator. So, the MM can provide a flexible and robust mechanism to process information fusion problems and more effectively solve MADM problems. However, in order to make the original MM operator process PFSs, it needs to be constrained to take only numeric arguments.

Although the IFSs theory has been applied in different fields, there are some real-life cases where IFSs are inappropriate. Voting can be a good example of this, because human voters can be divided into four groups: those who vote for, those who vote against, those who abstain, and those who refuse to vote. On the whole, PFSs [17] can handle human opinions that involve more answers, such as: yes, abstain, no, and refusal. However, none of the above methods is suitable for fusing picture fuzzy numbers (PFNs). Thus, the question of how to fuse PFN information is an interesting topic. In order to handle this case, in this article, we will present some picture fuzzy aggregation operators based on the traditional MM operators [57].

This research has four main purposes. The first is to develop a comprehensive MADM method for appraising financial investment risk with PFNs. The second lies in exploring several picture fuzzy aggregation operators based on the traditional MM operators. The third is to establish an integrated outranking decision-making method by the PFWMM (PFWDMM) operators. The final purpose is to demonstrate the application, practicality, and effectiveness of the proposed MADM method using a case study about financial investment risk.

For the sake of clarity, the rest of this research is organized as follows. Some basic definitions, operation rules, and score and accuracy functions of PFSs are introduced in the next section. Section 3 presents some picture fuzzy Muirhead mean aggregation operators, such as the PFMM operator; the picture fuzzy weighted MM (PFWMM) operator; the picture fuzzy dual MM (PFDMM) operator; and the picture fuzzy weighted dual (PFWDMM) operator. In Section 4, based on our defined aggregation operators and the PFN information, we build decision-making models to solve MADM problems. Section 5 gives a numerical example for evaluating a financial investment risk with picture fuzzy information in order to verify the method proposed in this article. Finally, some remarks are given to conclude this article.

\section{Preliminaries}

\subsection{Picture Fuzzy Sets}

Picture fuzzy sets (PFSs) [17], as the extension of intuitionistic fuzzy sets (IFSs) [1], have been considered to be an effective tool to depict uncertain information in the application of MADM problems. The basic definition and fundamental theory of PFSs are introduced as follows.

Definition $1([17,65])$. A PFS A on the domain $X$ is an object which denotes as:

$$
A=\left\{\left\langle x, \mu_{A}(x), \eta_{A}(x), v_{A}(x)\right\rangle \mid x \in X\right\}
$$

where $\mu_{A}(x) \in[0,1]$ is known as the positive-membership degree function, $\eta_{A}(x) \in[0,1]$ is known as the neutral-membership degree function, and $v_{A}(x) \in[0,1]$ is known as the negative-membership degree function. At the same time, for all $\forall x \in X, \mu_{A}(x), \eta_{A}(x)$ and $v_{A}(x)$ meet the following requirements: $0 \leq \mu_{A}(x)+\eta_{A}(x)+v_{A}(x) \leq 1$. Furthermore, the refusal-membership degree function is presented as $\pi_{A}(x)=1-\left(\mu_{A}(x)+\eta_{A}(x)+v_{A}(x)\right)$. For convenience, we call $\alpha=\left(\mu_{\alpha}, \eta_{\alpha}, v_{\alpha}\right)$ a PFN, where $\mu_{\alpha} \in$ $[0,1], \eta_{\alpha} \in[0,1], v_{\alpha} \in[0,1], \mu_{\alpha}+\eta_{\alpha}+v_{\alpha} \leq 1$. 
Definition 2 ([31]). Assume that $\alpha=\left(\mu_{\alpha}, \eta_{\alpha}, v_{\alpha}\right)$ and $\beta=\left(\mu_{\beta}, \eta_{\beta}, v_{\beta}\right)$ are two PFNs. The score function of $\alpha \beta$ can be denoted as $S(\alpha)=\mu_{\alpha}-v_{\alpha}$ and $S(\beta)=\mu_{\beta}-v_{\beta}$. Meanwhile, the accuracy functions of $\alpha$ and $\beta$ are presented as $H(\alpha)=\mu_{\alpha}+\eta_{\alpha}+v_{\alpha}$ and $H(\beta)=\mu_{\beta}+\eta_{\beta}+v_{\beta}$. Then, if $S(\alpha)<S(\beta), \alpha<\beta$; if $S(\alpha)=S(\beta)$, then

(1) If $H(a)=H(\beta), \alpha=\beta$;

(2) if $H(a)<H(\beta), \alpha<\beta$.

Similar to the IFS [66,67], Wei [31] has proposed some operational rules for PFNs.

Definition 3 ([31]). Assume that $\alpha=\left(\mu_{\alpha}, \eta_{\alpha}, v_{\alpha}\right)$ and $\beta=\left(\mu_{\beta}, \eta_{\beta}, v_{\beta}\right)$ are two PFNs. Then,

$$
\begin{aligned}
& \bar{\alpha}=\alpha=\left(v_{\alpha}, \eta_{\alpha}, \mu_{\alpha}\right) \\
& \alpha \wedge \beta=\left(\min \left\{\mu_{\alpha}, \mu_{\beta}\right\}, \max \left\{\eta_{\alpha}, \eta_{\beta}\right\}, \max \left\{v_{\alpha}, v_{\beta}\right\}\right) \\
& \alpha \vee \beta=\left(\max \left\{\mu_{\alpha}, \mu_{\beta}\right\}, \min \left\{\eta_{\alpha}, \eta_{\beta}\right\}, \min \left\{v_{\alpha}, v_{\beta}\right\}\right) \\
& \alpha \oplus \beta=\left(\mu_{\alpha}+\mu_{\beta}-\mu_{\alpha} \mu_{\beta}, \eta_{\alpha} \eta_{\beta}, v_{\alpha} v_{\beta}\right) ; \\
& \alpha \otimes \beta=\left(\mu_{\alpha} \mu_{\beta}, \eta_{\alpha}+\eta_{\beta}-\eta_{\alpha} \eta_{\beta}, v_{\alpha}+v_{\beta}-v_{\alpha} v_{\beta}\right) ; \\
& \lambda \alpha=\left(1-\left(1-\mu_{\alpha}\right)^{\lambda}, \eta_{\alpha}^{\lambda}, v_{\alpha}^{\lambda}\right) \\
& \alpha^{\lambda}=\left(\mu_{\alpha}^{\lambda}, 1-\left(1-\eta_{\alpha}\right)^{\lambda}, 1-\left(1-v_{\alpha}\right)^{\lambda}\right)
\end{aligned}
$$

According to Definition 3, Wei [31] obtained the following properties.

Theorem 1. Assume that $\alpha=\left(\mu_{\alpha}, \eta_{\alpha}, v_{\alpha}\right)$ and $\beta=\left(\mu_{\beta}, \eta_{\beta}, v_{\beta}\right)$ are two PFNs, $\lambda, \lambda_{1}, \lambda_{2}>0$, then

(1) $\alpha \oplus \beta=\beta \oplus \alpha ;$

(2) $\alpha \otimes \beta=\beta \otimes \alpha ;$

(3) $\lambda(\alpha \oplus \beta)=\lambda \alpha \oplus \lambda \beta$;

(4) $(\alpha \otimes \beta)^{\lambda}=\alpha^{\lambda} \otimes \beta^{\lambda}$;

(5) $\lambda_{1} \alpha \oplus \lambda_{2} \alpha=\left(\lambda_{1}+\lambda_{2}\right) \alpha$;

(6) $\alpha^{\lambda_{1}} \otimes \alpha^{\lambda_{2}}=\alpha^{\left(\lambda_{1}+\lambda_{2}\right)}$;

(7) $\left(\alpha^{\lambda_{1}}\right)^{\lambda_{2}}=\alpha^{\lambda_{1} \lambda_{2}}$.

\subsection{Operators}

Muirhead [57] proposed the Muirhead mean (MM) operator.

Definition 4 ([57]). Assume that $a_{j}(j=1,2, \ldots, n)$ is a set of non-negative real numbers, and let $P=$ $\left(p_{1}, p_{2}, \ldots, p_{n}\right) \in R^{n}$ be a vector of parameters. Then

$$
\operatorname{MM}^{\mathrm{P}}\left(a_{1}, a_{2}, \ldots, a_{n}\right)=\left(\frac{1}{n !} \sum_{\sigma \in S_{n}} \prod_{j=1}^{n} a_{\sigma(j)}^{p_{j}}\right)^{\sum_{j=1}^{n} p_{j}^{n}} .
$$

Then, we call $M M^{P}$ the Muirhead mean (MM) operator, where $\sigma(j)(j=1,2, \ldots, n)$ is any permutation of $\{1,2, \ldots, n\}$, and $S_{n}$ is the set of all permutations of $\{1,2, \ldots, n\}$.

\section{Picture Fuzzy Muirhead Mean Aggregation Operators}

In this part, based on PFN information and the MM operator, we are going to propose some new aggregation operators, including the PFMM operator and the PFWMM operator. 


\subsection{The PFMM Operator}

Definition 5. Assume that $\alpha_{j}=\left(\mu_{\alpha_{j}}, \eta_{\alpha_{j}}, v_{\alpha_{j}}\right)(j=1,2, \cdots, n)$ is a list of PFNs. The definition of the PFMM operator is expressed as:

$$
\operatorname{PFMM}^{\mathrm{P}}\left(\alpha_{1}, \alpha_{2}, \ldots, \alpha_{n}\right)=\left(\frac{1}{n !}\left(\bigoplus_{\sigma \in S_{n}}\left(\bigotimes_{j=1}^{n} \alpha_{\sigma(j)}^{p_{j}}\right)\right)\right)^{\frac{1}{\sum_{j=1}^{n} p_{j}}}
$$

Theorem 2. Assume that $\alpha_{j}=\left(\mu_{\alpha_{j}}, \eta_{\alpha_{j}}, v_{\alpha_{j}}\right)(j=1,2, \cdots, n)$ is a set of PFNs. We can fuse all the PFN information by utilizing the PFMM operator, and the fused results are shown as:

$$
\begin{aligned}
& \operatorname{PFMM}^{\mathrm{P}}\left(\alpha_{1}, \alpha_{2}, \ldots, \alpha_{n}\right)=\left(\frac{1}{n !}\left(\underset{\sigma \in S_{n}}{\oplus}\left(\underset{j=1}{\otimes} \alpha_{\sigma(j)}^{p_{j}}\right)\right)\right)^{\frac{1}{\sum_{j=1}^{n} p_{j}}} \\
& =\left\{\begin{array}{l}
\left(1-\prod_{\sigma \in S_{n}}\left(1-\prod_{j=1}^{n} \mu_{\alpha_{\sigma(j)}}^{p_{j}}\right)^{\frac{1}{n !}}\right)^{\frac{1}{\sum_{j=1}^{n} p_{j}}}, 1-\left(1-\prod_{\sigma \in S_{n}}\left(1-\prod_{j=1}^{n}\left(1-\eta_{\alpha_{\sigma(j)}}\right)^{p_{j}}\right)^{\frac{1}{n !}}\right)^{\frac{1}{\sum_{j=1}^{n} p_{j}}}, \\
1-\left(1-\prod_{\sigma \in S_{n}}\left(1-\prod_{j=1}^{n}\left(1-v_{\alpha_{\sigma(j)}}\right)^{p_{j}}\right)^{\frac{1}{n !}}\right)^{\frac{1}{\sum_{j=1}^{n} p_{j}}}
\end{array}\right\}
\end{aligned}
$$

Proof.

$$
\alpha_{\sigma(j)}^{p_{j}}=\left\{\mu_{\alpha_{\sigma(j)}}^{p_{j}}, 1-\left(1-\eta_{\alpha_{\sigma(j)}}\right)^{p_{j}}, 1-\left(1-v_{\alpha_{\sigma(j)}}\right)^{p_{j}}\right\}
$$

Thus,

$$
\bigotimes_{j=1}^{n} \alpha_{\sigma(j)}^{p_{j}}=\left\{\prod_{j=1}^{n} \mu_{\alpha_{\sigma(j)}}^{p_{j}}, 1-\prod_{j=1}^{n}\left(1-\eta_{\alpha_{\sigma(j)}}\right)^{p_{j}}, 1-\prod_{j=1}^{n}\left(1-v_{\alpha_{\sigma(j)}}\right)^{p_{j}}\right\} .
$$

Thereafter,

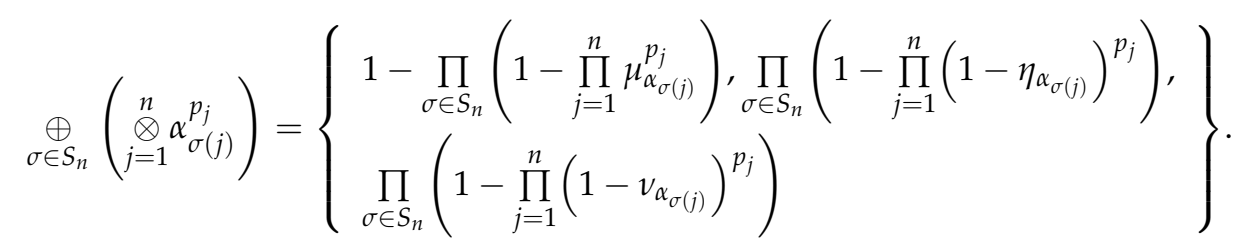

Furthermore,

$$
\frac{1}{n !}\left(\underset{\sigma \in S_{n}}{\oplus}\left(\otimes_{j=1}^{n} \alpha_{\sigma(j)}^{p_{j}}\right)\right)=\left\{\begin{array}{l}
\left(1-\prod_{\sigma \in S_{n}}\left(1-\prod_{j=1}^{n} \mu_{\alpha_{\sigma(j)}}^{p_{j}}\right)^{\frac{1}{n !}}\right), \prod_{\sigma \in S_{n}}\left(1-\prod_{j=1}^{n}\left(1-\eta_{\alpha_{\sigma(j)}}\right)^{p_{j}}\right)^{\frac{1}{n !}}, \\
\prod_{\sigma \in S_{n}}\left(1-\prod_{j=1}^{n}\left(1-v_{\alpha_{\sigma(j)}}\right)^{p_{j}}\right)^{\frac{1}{n !}}
\end{array}\right\} .
$$

Therefore, 


$$
\begin{aligned}
& \left.\operatorname{PFMM}^{\mathrm{P}}\left(\alpha_{1}, \alpha_{2}, \ldots, \alpha_{n}\right)=\left(\frac{1}{n !}\left(\underset{\sigma \in S_{n}}{\oplus} \underset{j=1}{\stackrel{n}{\otimes}} \alpha_{\sigma(j)}^{p_{j}}\right)\right)\right)^{\frac{1}{\sum_{j=1}^{n} p_{j}}} \\
& =\left\{\begin{array}{l}
\left(1-\prod_{\sigma \in S_{n}}\left(1-\prod_{j=1}^{n} \mu_{\alpha_{\sigma(j)}}^{p_{j}}\right)^{\frac{1}{n !}}\right)^{\sum_{j=1}^{n} p_{j}}, 1-\left(1-\prod_{\sigma \in S_{n}}\left(1-\prod_{j=1}^{n}\left(1-\eta_{\alpha_{\sigma(j)}}\right)^{p_{j}}\right)^{\frac{1}{n !}}\right)^{\frac{1}{\Sigma_{j=1}^{n} p_{j}}}, \\
1-\left(1-\prod_{\sigma \in S_{n}}\left(1-\prod_{j=1}^{n}\left(1-v_{\alpha_{\sigma(j)}}\right)^{p_{j}}\right)^{\frac{1}{n !}}\right)^{\frac{1}{\sum_{j=1}^{n} p_{j}}}
\end{array}\right\}
\end{aligned}
$$

Hence, (4) is kept.

Example 1. Let $(0.43,0.36,0.19),(0.79,0.02,0.10),(0.44,0.45,0.08)$ be three PFNs, and $\mathrm{P}=(0.2,0.3,0.5)$, $\sum_{j=1}^{3} p_{j}=1$. Then, according to (4), we have

$$
\begin{aligned}
& \operatorname{PFMM}^{(0.2,0.3,3.5)}((0.43,0.36,0.19),(0.79,0.02,0.10),(0.44,0.45,0.08))
\end{aligned}
$$

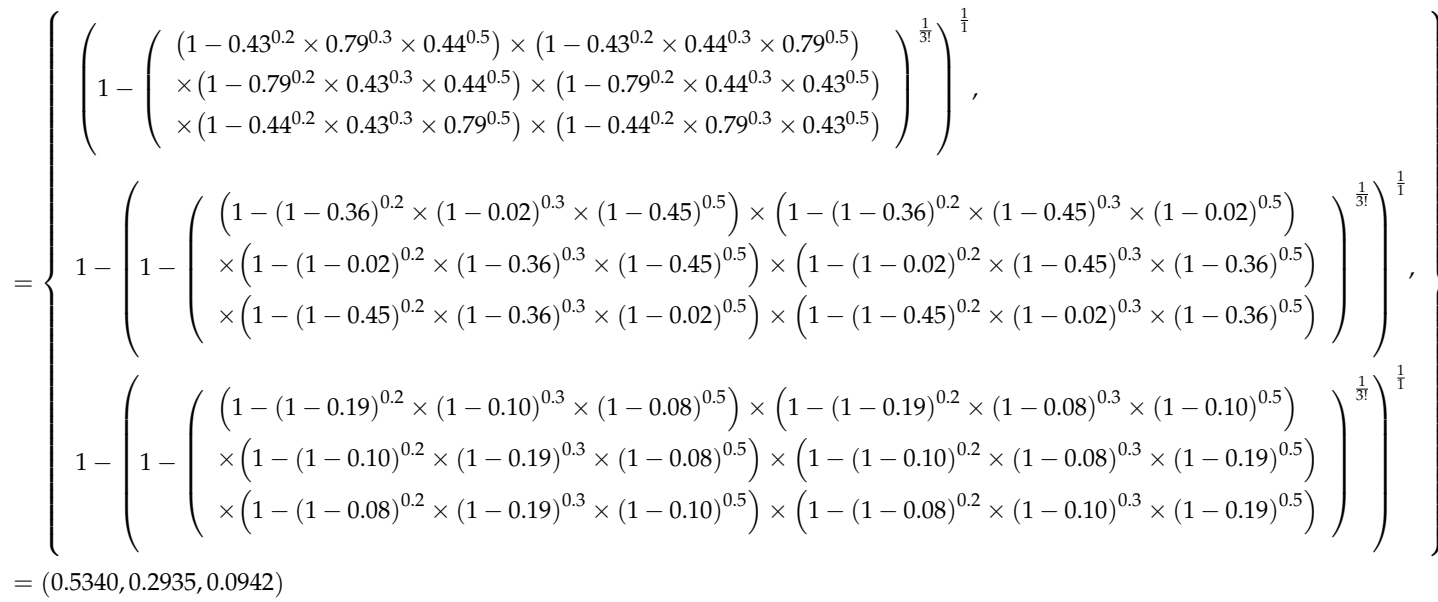

It is clear that the PFMM operator satisfies the following three properties.

Theorem 3 (Idempotency). If all $\alpha_{j}(j=1,2, \cdots, n)$ are equal, i.e., $\alpha_{j}=\alpha$ for all $j$, then

$$
\operatorname{PFMM}^{\mathrm{P}}\left(\alpha_{1}, \alpha_{2}, \cdots, \alpha_{n}\right)=\alpha
$$

Theorem 4 (Boundedness). Assume that $\alpha_{j}(j=1,2, \cdots, n)$ is a group of PFNs. If

$$
\alpha^{-}=\min _{j} \alpha_{j}, \alpha^{+}=\max _{j} \alpha_{j}
$$

Then

$$
\alpha^{-} \leq \operatorname{PFMM}^{\mathrm{P}}\left(\alpha_{1}, \alpha_{2}, \cdots, \alpha_{n}\right) \leq \alpha^{+}
$$

Theorem 5 (Monotonicity). Assume that $\alpha_{j}(j=1,2, \cdots, n)$ and $\alpha^{\prime}{ }_{j}(j=1,2, \cdots, n)$ are two lists of PFNs. Let $\alpha_{j} \leq \alpha^{\prime}{ }_{j}$ for all $j$. Then,

$$
\operatorname{PFMM}^{\mathrm{P}}\left(\alpha_{1}, \alpha_{2}, \cdots, \alpha_{n}\right) \leq \operatorname{PFMM}^{\mathrm{P}}\left(\alpha^{\prime}{ }_{1}, \alpha^{\prime}{ }_{2}, \cdots, \alpha_{n}^{\prime}\right) .
$$




\subsection{The PFWMM Operator}

To take an attribute's weight into account, the picture fuzzy weighted MM (PFWMM) operator can be defined as follows.

Definition 6. Assume that $\alpha_{j}=\left(\mu_{\alpha_{j}}, \eta_{\alpha_{j}}, v_{\alpha_{j}}\right)(j=1,2, \cdots, n)$ is a list of PFNs. The PFWMM operator can be defined as:

$$
\operatorname{PFWMM}_{n w}^{P}\left(\alpha_{1}, \alpha_{2}, \ldots, \alpha_{n}\right)=\left(\frac{1}{n !}\left(\underset{\sigma \in S_{n}}{\bigoplus}\left(\bigotimes_{j=1}^{n}\left(n w_{\sigma(j)} \alpha_{\sigma(j)}\right)\right)^{p_{j}}\right)\right)^{\frac{1}{\sum_{j=1}^{n} p_{j}}}
$$

Theorem 6. Assume that $\alpha_{j}=\left(\mu_{\alpha_{j}}, \eta_{\alpha_{j}}, v_{\alpha_{j}}\right)(j=1,2, \cdots, n)$ is a group of PFNs. We can fuse all the PFN information by utilizing the PFWMM operator, and the fused results are shown as:

$$
\begin{aligned}
& \operatorname{PFWMM}_{n w}^{P}=\left(\frac{1}{n !}\left(\underset{\sigma \in S_{n}}{\oplus}\left(\bigotimes_{j=1}^{n}\left(n w_{\sigma(j)} \alpha_{\sigma(j)}\right)\right)\right)^{p_{j}}\right)^{\frac{1}{\sum_{j=1}^{n} p_{j}}} \\
& =\left\{\begin{array}{c}
\left(1-\prod_{\sigma \in S_{n}}\left(1-\prod_{j=1}^{n}\left(1-\left(1-\mu_{\alpha_{\sigma(j)}}\right)^{n w w_{\sigma(j)}}\right)^{p_{j}}\right)^{\frac{1}{n !}}\right)^{\frac{1}{\Sigma_{j=1}^{n} p_{j}}}, \\
1-\left(1-\prod_{\sigma \in S_{n}}\left(1-\prod_{j=1}^{n}\left(1-\left(\eta_{\alpha_{\sigma(j)}}\right)^{n w_{\sigma(j)}}\right)^{p_{j}}\right)^{\frac{1}{n !}}\right)^{\frac{1}{\Sigma_{j=1}^{n} p_{j}}}, \\
1-\left(1-\prod_{\sigma \in S_{n}}\left(1-\prod_{j=1}^{n}\left(1-\left(v_{\alpha_{\sigma(j)}}\right)^{n w w_{\sigma(j)}}\right)^{p_{j}}\right)^{\frac{1}{n !}}\right)^{\frac{1}{\Sigma_{j=1}^{n} p_{j}}}
\end{array}\right\}
\end{aligned}
$$

\section{Proof.}

$$
n w_{\sigma(j)} \alpha_{\sigma(j)}=\left\{1-\left(1-\mu_{\alpha_{\sigma(j)}}\right)^{n w_{\sigma(j)}},\left(\eta_{\alpha_{\sigma(j)}}\right)^{n w_{\sigma(j)}},\left(v_{\alpha_{\sigma(j)}}\right)^{n w_{\sigma(j)}}\right\}
$$

Thus,

$$
\left(n w_{\sigma(j)} \alpha_{\sigma(j)}\right)^{p_{j}}=\left\{\left(1-\left(1-\mu_{\alpha_{\sigma(j)}}\right)^{n w w_{\sigma(j)}}\right)^{p_{j}}, 1-\left(1-\left(\eta_{\alpha_{\sigma(j)}}\right)^{n w w_{\sigma(j)}}\right)^{p_{j}}, 1-\left(1-\left(v_{\alpha_{\sigma(j)}}\right)^{n w_{\sigma(j)}}\right)^{p_{j}}\right\} .
$$

Therefore,

$$
\bigotimes_{j=1}^{n}\left(n w_{\sigma(j)} \alpha_{\sigma(j)}\right)^{p_{j}}=\left\{\begin{array}{l}
\prod_{j=1}^{n}\left(1-\left(1-\mu_{\alpha_{\sigma(j)}}\right)^{n w_{\sigma(j)}}\right)^{p_{j}}, 1-\prod_{j=1}^{n}\left(1-\left(\eta_{\alpha_{\sigma(j)}}\right)^{n w_{\sigma(j)}}\right)^{p_{j}}, \\
1-\prod_{j=1}^{n}\left(1-\left(v_{\alpha_{\sigma(j)}}\right)^{n w_{\sigma(j)}}\right)^{p_{j}}
\end{array}\right\} .
$$


Thereafter,

$$
\begin{aligned}
& \underset{\sigma \in S_{n}}{\oplus}\left(\bigotimes_{j=1}^{n}\left(n w_{\sigma(j)} \alpha_{\sigma(j)}\right)^{p_{j}}\right) \\
& =\left\{\begin{array}{l}
1-\prod_{\sigma \in S_{n}}\left(1-\prod_{j=1}^{n}\left(1-\left(1-\mu_{\alpha_{\sigma(j)}}\right)^{n w_{\sigma(j)}}\right)^{p_{j}}\right), \prod_{\sigma \in S_{n}}\left(1-\prod_{j=1}^{n}\left(1-\left(\eta_{\alpha_{\sigma(j)}}\right)^{n w_{\sigma(j)}}\right)^{p_{j}}\right), \\
\prod_{\sigma \in S_{n}}\left(1-\prod_{j=1}^{n}\left(1-\left(v_{\alpha_{\sigma(j)}}\right)^{n w_{\sigma(j)}}\right)^{p_{j}}\right.
\end{array}\right\}
\end{aligned}
$$

Furthermore,

$$
\begin{aligned}
& \frac{1}{n !}\left(\underset{\sigma \in S_{n}}{\oplus}\left({\underset{j=1}{\otimes}}_{j=1}^{n}\left(n w_{\sigma(j)} \alpha_{\sigma(j)}\right)^{p_{j}}\right)\right) \\
& =\left\{\begin{array}{c}
1-\prod_{\sigma \in S_{n}}\left(1-\prod_{j=1}^{n}\left(1-\left(1-\mu_{\alpha_{\sigma(j)}}\right)^{n w_{\sigma(j)}}\right)^{p_{j}}\right)^{\frac{1}{n !}}, \prod_{\sigma \in S_{n}}\left(1-\prod_{j=1}^{n}\left(1-\left(\eta_{\alpha_{\sigma(j)}}\right)^{n w_{\sigma(j)}}\right)^{p_{j}}\right)^{\frac{1}{n !}}, \\
\prod_{\sigma \in S_{n}}\left(1-\prod_{j=1}^{n}\left(1-\left(v_{\alpha_{\sigma(j)}}\right)^{n w_{\sigma(j)}}\right)^{p_{j}}\right)^{\frac{1}{n !}}
\end{array}\right\}
\end{aligned}
$$

Therefore,

$$
\begin{aligned}
& \operatorname{PFWMM}_{n w}^{P}=\left(\frac{1}{n !}\left(\bigoplus_{\sigma \in S_{n}}\left(\prod_{j=1}^{n}\left(n w_{\sigma(j)} \alpha_{\sigma(j)}\right)^{p_{j}}\right)\right)\right)^{\frac{1}{\sum_{j=1}^{n} p_{j}}} \\
& =\left\{\begin{array}{l}
\left(1-\prod_{\sigma \in S_{n}}\left(1-\prod_{j=1}^{n}\left(1-\left(1-\mu_{\alpha_{\sigma(j)}}\right)^{n w w_{\sigma(j)}}\right)^{p_{j}}\right)^{\frac{1}{n !}}\right)^{\frac{1}{\sum_{j=1}^{n} p_{j}}}, \\
1-\left(1-\prod_{\sigma \in S_{n}}\left(1-\prod_{j=1}^{n}\left(1-\left(\eta_{\alpha_{\sigma(j)}}\right)^{n w_{\sigma(j)}}\right)^{p_{j}}\right)^{\frac{1}{n !}}\right)^{\frac{1}{\sum_{j=1}^{n} p_{j}}}, \\
1-\left(1-\prod_{\sigma \in S_{n}}\left(1-\prod_{j=1}^{n}\left(1-\left(v_{\alpha_{\sigma(j)}}\right)^{n w_{\sigma(j)}}\right)^{p_{j}}\right)^{\frac{1}{n !}}\right)^{\frac{1}{\sum_{j=1}^{n} p_{j}}}
\end{array}\right\}
\end{aligned}
$$

Hence, (14) is kept.

Example 2. Let $(0.43,0.36,0.19),(0.79,0.02,0.10),(0.44,0.45,0.08)$ be three PFNs, and $\mathrm{P}=(0.2,0.3,0.5)$, $\sum_{j=1}^{3} p_{j}=1$ and $w=(0.4,0.3,0.3)$. Then, according to (4), we have 


$$
\begin{aligned}
& \operatorname{PFWMM}_{(0.4,0.3,0.3)}^{(0.2,0.3,0)}((0.43,0.36,0.19),(0.79,0.02,0.10),(0.44,0.45,0.08))
\end{aligned}
$$

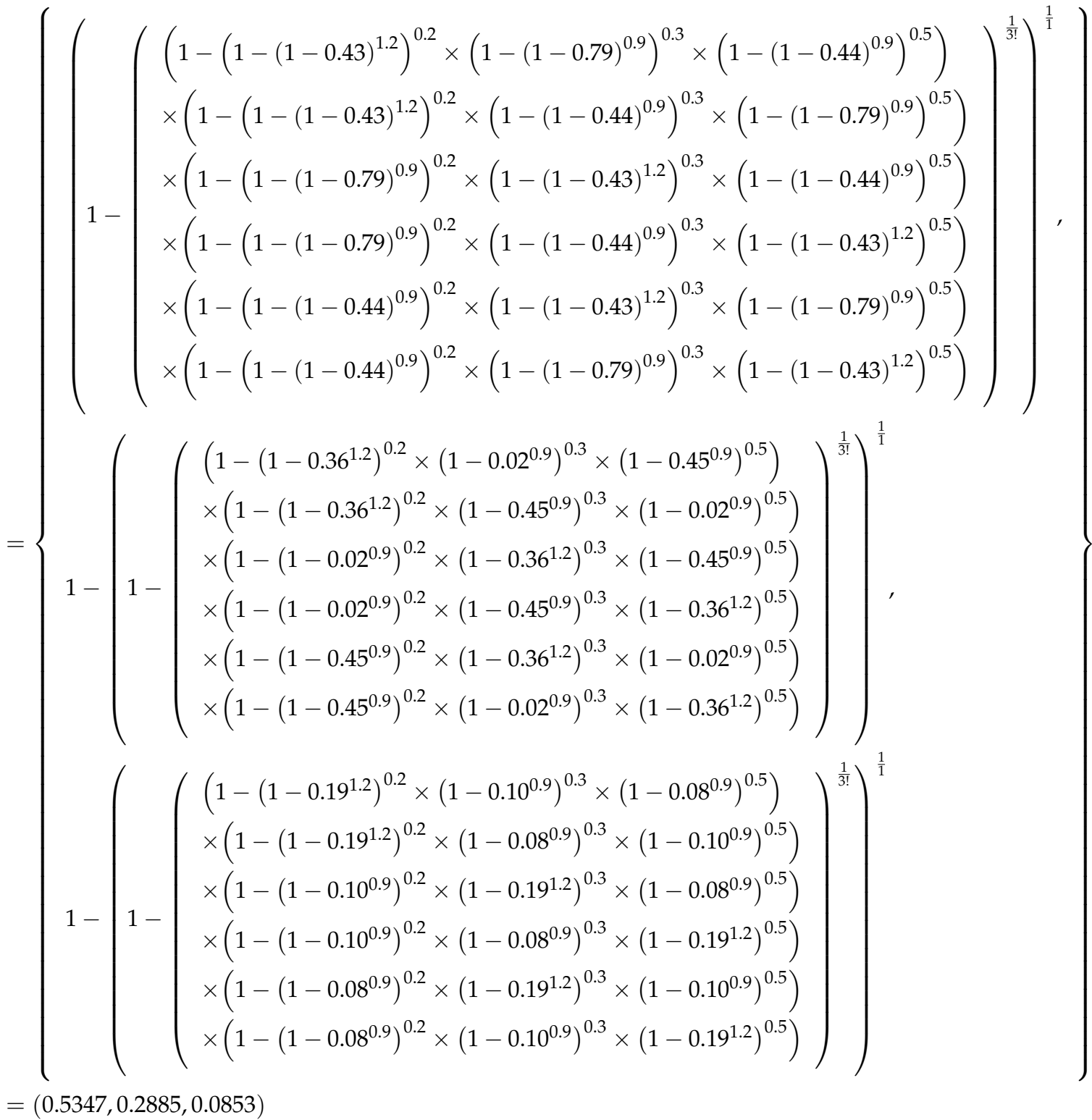

It is clear that the PFWMM operator satisfies the following two properties.

Theorem 7 (Boundedness). Assume that $\alpha_{j}(j=1,2, \cdots, n)$ is a group of PFNs. If

$$
\alpha^{-}=\min _{j} \alpha_{j}, \alpha^{+}=\max _{j} \alpha_{j}
$$

Then

$$
\alpha^{-} \leq \operatorname{PFWMM}_{n w}^{P}\left(\alpha_{1}, \alpha_{2}, \cdots, \alpha_{n}\right) \leq \alpha^{+}
$$

Theorem 8 (Monotonicity). Assume that $\alpha_{j}(j=1,2, \cdots, n)$ and $\alpha^{\prime}{ }_{j}(j=1,2, \cdots, n)$ are two groups of PFNs. Let $\alpha_{j} \leq \alpha^{\prime}{ }_{j}$ for all $j$. Then,

$$
\operatorname{PFWMM}_{n w}^{P}\left(\alpha_{1}, \alpha_{2}, \cdots, \alpha_{n}\right) \leq \operatorname{PFWMM}_{n w}^{P}\left(\alpha^{\prime}{ }_{1}, \alpha^{\prime}{ }_{2}, \cdots, \alpha^{\prime}{ }_{n}\right) .
$$




\subsection{The PFDMM Operator}

Qin and Liu [68] proposed the dual MM (DMM) as follows.

Definition 7 ([68]). Assume that $a_{i}(i=1,2, \ldots, n)$ is a set of non-negative real numbers, and let $P=$ $\left(p_{1}, p_{2}, \ldots, p_{n}\right) \in R^{n}$ be a vector of parameters. Then,

$$
\operatorname{DMM}^{\mathrm{P}}\left(a_{1}, a_{2}, \ldots, a_{n}\right)=\frac{1}{\sum_{j=1}^{n} p_{j}}\left(\prod_{\sigma \in S_{n}} \sum_{j=1}^{n} p_{j} a_{\sigma(j)}\right)^{\frac{1}{n !}} .
$$

Then, we call $\mathrm{DMM}^{\mathrm{P}}$ the dual MM (DMM) operator, where $\sigma(j)(j=1,2, \ldots, n)$ is any permutation of $\{1,2, \ldots, n\}$, and $S_{n}$ is the set of all permutations of $\{1,2, \ldots, n\}$.

Combining the PFN information and the DMM operator, the definition of the PFDMM operator can be developed as follows.

Definition 8. Assume that $\alpha_{j}=\left(\mu_{\alpha_{j}}, \eta_{\alpha_{j}}, v_{\alpha_{j}}\right)(j=1,2, \cdots, n)$ is a group of PFNs, and let $P=$ $\left(p_{1}, p_{2}, \ldots, p_{n}\right) \in R^{n}$ be a vector of parameters. Then,

$$
\operatorname{PFDMM}^{P}\left(\alpha_{1}, \alpha_{2}, \ldots, \alpha_{j}\right)=\frac{1}{\sum_{j=1}^{n} p_{j}}\left(\underset{\sigma \in S_{n}}{\otimes}\left(\bigoplus_{j=1}^{n}\left(p_{j} \alpha_{\sigma(j)}\right)\right)\right)^{\frac{1}{n !}} .
$$

Then, we call $\operatorname{PFDMM}^{P}$ the picture fuzzy DMM (PFDMM) operator, where $\sigma(j)(j=1,2, \ldots, n)$ is any permutation of $\{1,2, \ldots, n\}$, and $S_{n}$ is the set of all permutations of $\{1,2, \ldots, n\}$.

Theorem 9. Assume that $\alpha_{j}=\left(\mu_{\alpha_{j}}, \eta_{\alpha_{j}}, v_{\alpha_{j}}\right)(j=1,2, \cdots, n)$ is a list of PFNs. We can fuse all the PFN information by utilizing the PFDMM operator, and the fused results are shown as:

$$
\begin{aligned}
& \operatorname{PFDMM}^{P}\left(\alpha_{1}, \alpha_{2}, \ldots, \alpha_{n}\right)=\frac{1}{\sum_{j=1}^{n} p_{j}}\left(\underset{\sigma \in S_{n}}{\otimes}\left(\bigoplus_{j=1}^{n}\left(p_{j} \alpha_{\sigma(j)}\right)\right)\right)^{\frac{1}{n !}} \\
& =\left\{\begin{array}{l}
1-\left(1-\prod_{\sigma \in S_{n}}\left(1-\prod_{j=1}^{n}\left(1-\mu_{\alpha_{j}}\right)^{p_{j}}\right)^{\frac{1}{n !}}\right)^{\frac{1}{\sum_{j=1}^{n} p_{j}}},\left(1-\prod_{\sigma \in S_{n}}\left(1-\prod_{j=1}^{n}\left(\eta_{\alpha_{j}}\right)^{p_{j}}\right)^{\frac{1}{n !}}\right)^{\frac{1}{\sum_{j=1}^{n} p_{j}}}, \\
\left(1-\prod_{\sigma \in S_{n}}\left(1-\prod_{j=1}^{n}\left(v_{\alpha_{j}}\right)^{p_{j}}\right)^{\frac{1}{n !}}\right)^{\frac{1}{\sum_{j=1}^{n} p_{j}}}
\end{array}\right\}
\end{aligned}
$$

Proof.

$$
p_{j} \alpha_{\sigma(j)}=\left\{1-\left(1-\mu_{\alpha_{j}}\right)^{p_{j}},\left(\eta_{\alpha_{j}}\right)^{p_{j}},\left(v_{\alpha_{j}}\right)^{p_{j}}\right\}
$$

Thus,

$$
\bigoplus_{j=1}^{n}\left(p_{j} \alpha_{\sigma(j)}\right)=\left\{1-\prod_{j=1}^{n}\left(1-\mu_{\alpha_{j}}\right)^{p_{j}}, \prod_{j=1}^{n}\left(\eta_{\alpha_{j}}\right)^{p_{j}}, \prod_{j=1}^{n}\left(v_{\alpha_{j}}\right)^{p_{j}}\right\} .
$$

Therefore, 


$$
\underset{\sigma \in S_{n}}{\otimes}\left(\stackrel{\oplus}{j=1}_{j=1}^{n}\left(p_{j} \alpha_{\sigma(j)}\right)\right)=\left\{\begin{array}{l}
\prod_{\sigma \in S_{n}}\left(1-\prod_{j=1}^{n}\left(1-\mu_{\alpha_{j}}\right)^{p_{j}}\right), 1-\prod_{\sigma \in S_{n}}\left(1-\prod_{j=1}^{n}\left(\eta_{\alpha_{j}}\right)^{p_{j}}\right) \\
1-\prod_{\sigma \in S_{n}}\left(1-\prod_{j=1}^{n}\left(v_{\alpha_{j}}\right)^{p_{j}}\right)
\end{array}\right\} .
$$

\section{Furthermore,}

$$
\begin{aligned}
& \left(\underset{\sigma \in S_{n}}{\otimes}\left(\bigoplus_{j=1}^{n}\left(p_{j} \alpha_{\sigma(j)}\right)\right)\right)^{\frac{1}{n !}} \\
& =\left\{\begin{array}{l}
\prod_{\sigma \in S_{n}}\left(1-\prod_{j=1}^{n}\left(1-\mu_{\alpha_{j}}\right)^{p_{j}}\right)^{\frac{1}{n !}}, 1-\prod_{\sigma \in S_{n}}\left(1-\prod_{j=1}^{n}\left(\eta_{\alpha_{j}}\right)^{p_{j}}\right)^{\frac{1}{n !}}, \\
1-\prod_{\sigma \in S_{n}}\left(1-\prod_{j=1}^{n}\left(v_{\alpha_{j}}\right)^{p_{j}}\right)^{\frac{1}{n !}}
\end{array}\right\}
\end{aligned}
$$

Therefore,

$$
\begin{aligned}
& \operatorname{PFDMM}^{P}\left(\alpha_{1}, \alpha_{2}, \ldots, \alpha_{n}\right)=\frac{1}{\sum_{j=1}^{n} p_{j}}\left(\underset{\sigma \in S_{n}}{\otimes}\left({\underset{j=1}{\oplus}}_{j}^{n}\left(p_{j} \alpha_{\sigma(j)}\right)\right)\right)^{\frac{1}{n !}} \\
& =\left\{\begin{array}{l}
1-\left(1-\prod_{\sigma \in S_{n}}\left(1-\prod_{j=1}^{n}\left(1-\mu_{\alpha_{j}}\right)^{p_{j}}\right)^{\frac{1}{n !}}\right)^{\frac{1}{\sum_{j=1}^{n} p_{j}}},\left(1-\prod_{\sigma \in S_{n}}\left(1-\prod_{j=1}^{n}\left(\eta_{\alpha_{j}}\right)^{p_{j}}\right)^{\frac{1}{n !}}\right)^{\frac{1}{\sum_{j=1}^{n} p_{j}}}, \\
\left(1-\prod_{\sigma \in S_{n}}\left(1-\prod_{j=1}^{n}\left(v_{\alpha_{j}}\right)^{p_{j}}\right)^{\frac{1}{n !}}\right)^{\frac{1}{\sum_{j=1}^{n} p_{j}}}
\end{array}\right\}
\end{aligned}
$$

Hence, (25) is kept.

Example 3. Let $(0.43,0.36,0.19),(0.79,0.02,0.10),(0.44,0.45,0.08)$ be three PFNs, and $\mathrm{P}=(0.2,0.3,0.5)$, $\sum_{j=1}^{3} p_{j}=1$. Then, according to (25), we have

$$
\begin{aligned}
& \operatorname{PFDMM}^{(0.2,0.3,0.5)}((0.43,0.36,0.19),(0.79,0.02,0.10),(0.44,0.45,0.08)) \\
& \left.\left.\begin{array}{l}
1-\left(1-\left(\begin{array}{l}
\left(1-(1-0.43)^{0.2} \times(1-0.79)^{0.3} \times(1-0.44)^{0.5}\right) \times\left(1-(1-0.43)^{0.2} \times(1-0.44)^{0.3} \times(1-0.79)^{0.5}\right) \\
\times\left(1-(1-0.02)^{0.2} \times(1-0.43)^{0.3} \times(1-0.44)^{0.5}\right) \times\left(1-(1-0.79)^{0.2} \times(1-0.44)^{0.3} \times(1-0.43)^{0.5}\right) \\
\times\left(1-(1-0.44)^{0.2} \times(1-0.43)^{0.3} \times(1-0.79)^{0.5}\right) \times\left(1-(1-0.44)^{0.2} \times(1-0.79)^{0.3} \times(1-0.43)^{0.5}\right)
\end{array}\right)\right.
\end{array}\right)\right\} \\
& =(0.5887,0.1598,0.0563)
\end{aligned}
$$

It is clear that the PFDMM operator satisfies the following three properties. 
Theorem 10 (Idempotency). Let $\alpha_{j}(j=1,2, \cdots, n)$ be equal, i.e., $\alpha_{j}=\alpha$ for all $j$. Then,

$$
\operatorname{PFDMM}^{\mathrm{P}}\left(\alpha_{1}, \alpha_{2}, \cdots, \alpha_{n}\right)=\alpha .
$$

Theorem 11 (Boundedness). Assume that $\alpha_{j}(j=1,2, \cdots, n)$ is a list of PFNs. If

$$
\alpha^{-}=\min _{j} \alpha_{j}, \alpha^{+}=\max _{j} \alpha_{j}
$$

Then

$$
\alpha^{-} \leq \operatorname{PFDMM}^{\mathrm{P}}\left(\alpha_{1}, \alpha_{2}, \cdots, \alpha_{n}\right) \leq \alpha^{+} .
$$

Theorem 12 (Monotonicity). Assume that $\alpha_{j}(j=1,2, \cdots, n)$ and $\alpha^{\prime}{ }_{j}(j=1,2, \cdots, n)$ are two groups of PFNs, and let $\alpha_{j} \leq \alpha^{\prime}{ }_{j}$ for all $j$. Then,

$$
\operatorname{PFDMM}^{\mathrm{P}}\left(\alpha_{1}, \alpha_{2}, \cdots, \alpha_{n}\right) \leq \operatorname{PFDMM}^{\mathrm{P}}\left(\alpha^{\prime}{ }_{1}, \alpha^{\prime}{ }_{2}, \cdots, \alpha^{\prime}{ }_{n}\right) .
$$

\subsection{The PFWDMM Operator}

To take an attribute's weight into account, the picture fuzzy weighted DMM (PFWDMM) operator can be defined as follows.

Definition 9. Assume that $\alpha_{j}=\left(\mu_{\alpha_{j}}, \eta_{\alpha_{j}}, v_{\alpha_{j}}\right)(j=1,2, \cdots, n)$ is a set of PFNs. The definition of the PFWDMM operator can be expressed as:

$$
\operatorname{PFWDMM}_{n w}^{P}\left(\alpha_{1}, \alpha_{2}, \cdots, \alpha_{n}\right)=\frac{1}{\sum_{j=1}^{n} p_{j}}\left(\underset{\sigma \in S_{n}}{\otimes}\left(\bigoplus_{j=1}^{n}\left(p_{j} \alpha_{\sigma(j)}^{n w_{\sigma(j)}}\right)\right)\right)^{\frac{1}{n !}} .
$$

Theorem 13. Assume that $\alpha_{j}=\left(\mu_{\alpha_{j}}, \eta_{\alpha_{j}}, v_{\alpha_{j}}\right)(j=1,2, \cdots, n)$ is a group of PFNs. We can fuse all the PFN information by utilizing the PFWDMM operator, and the fused results are shown as:

$$
\begin{aligned}
& \operatorname{PFWDMM}_{n w}^{P}\left(\alpha_{1}, \alpha_{2}, \cdots, \alpha_{n}\right) \\
& =\frac{1}{\sum_{j=1}^{n} p_{j}} \underset{\sigma \in S_{n}}{\otimes}\left(\bigoplus_{j=1}^{n}\left(p_{j} \alpha_{\sigma(j)}^{\left.n w_{\sigma(j)}\right)}\right)\right)^{\frac{1}{n !}} \\
& =\left\{\begin{array}{c}
1-\left(1-\prod_{\sigma \in S_{n}}\left(1-\prod_{j=1}^{n}\left(1-\left(\mu_{\alpha_{j}}\right)^{n w_{\sigma(j)}}\right)^{p_{j}}\right)^{\frac{1}{n !}}\right)^{\frac{1}{\sum_{j=1}^{n} p_{j}}}, \\
\left(1-\prod_{\sigma \in S_{n}}\left(1-\prod_{j=1}^{n}\left(1-\left(1-\eta_{\alpha_{j}}\right)^{n w_{\sigma(j)}}\right)^{p_{j}}\right)^{\frac{1}{n !}}\right)^{\frac{1}{\sum_{j=1}^{n} p_{j}}}, \\
\left(1-\prod_{\sigma \in S_{n}}\left(1-\prod_{j=1}^{n}\left(1-\left(1-v_{\alpha_{j}}\right)^{n w w_{\sigma(j)}}\right)^{p_{j}}\right)^{\frac{1}{n !}}\right)^{\frac{\sum_{j=1}^{n} p_{j}}{n}}
\end{array}\right\}
\end{aligned}
$$

Proof.

$$
\alpha_{\sigma(j)}^{n w_{\sigma(j)}}=\left\{\left(\mu_{\alpha_{j}}\right)^{n w w_{\sigma(j)}}, 1-\left(1-\eta_{\alpha_{j}}\right)^{n w_{\sigma(j)}}, 1-\left(1-v_{\alpha_{j}}\right)^{n w_{\sigma(j)}}\right\}
$$


Then,

$$
p_{j} \alpha_{\sigma(j)}^{n w w_{\sigma(j)}}=\left\{1-\left(1-\left(\mu_{\alpha_{j}}\right)^{n w_{\sigma(j)}}\right)^{p_{j}},\left(1-\left(1-\eta_{\alpha_{j}}\right)^{n w_{\sigma(j)}}\right)^{p_{j}},\left(1-\left(1-v_{\alpha_{j}}\right)^{n w w_{\sigma(j)}}\right)^{p_{j}}\right\} .
$$

Thus,

$$
\bigoplus_{j=1}^{n}\left(p_{j} \alpha_{\sigma(j)}^{n w w_{\sigma(j)}}\right)=\left\{\begin{array}{l}
1-\prod_{j=1}^{n}\left(1-\left(\mu_{\alpha_{j}}\right)^{n w_{\sigma(j)}}\right)^{p_{j}}, \prod_{j=1}^{n}\left(1-\left(1-\eta_{\alpha_{j}}\right)^{n w w_{\sigma(j)}}\right)^{p_{j}}, \\
\prod_{j=1}^{n}\left(1-\left(1-v_{\alpha_{j}}\right)^{n w_{\sigma(j)}}\right)^{p_{j}}
\end{array}\right\} .
$$

Therefore,

$$
\begin{aligned}
& \underset{\sigma \in S_{n}}{\otimes}\left(\bigoplus_{j=1}^{n}\left(p_{j} \alpha_{\sigma(j)}^{n w w_{\sigma(j)}}\right)\right) \\
& =\left\{\begin{array}{l}
\prod_{\sigma \in S_{n}}\left(1-\prod_{j=1}^{n}\left(1-\left(\mu_{\alpha_{j}}\right)^{n w_{\sigma(j)}}\right)^{p_{j}}\right), 1-\prod_{\sigma \in S_{n}}\left(1-\prod_{j=1}^{n}\left(1-\left(1-\eta_{\alpha_{j}}\right)^{n w_{\sigma(j)}}\right)^{p_{j}}\right), \\
1-\prod_{\sigma \in S_{n}}\left(1-\prod_{j=1}^{n}\left(1-\left(1-v_{\alpha_{j}}\right)^{n w w_{\sigma(j)}}\right)^{p_{j}}\right.
\end{array}\right\}
\end{aligned}
$$

Furthermore,

$$
\begin{aligned}
& \underset{\sigma \in S_{n}}{\otimes}\left(\prod_{j=1}^{n}\left(p_{j} \alpha_{\sigma(j)}^{n w_{\sigma(j)}}\right)\right)^{\frac{1}{n !}} \\
& =\left\{\begin{array}{l}
\prod_{\sigma \in S_{n}}\left(1-\prod_{j=1}^{n}\left(1-\left(\mu_{\alpha_{j}}\right)^{n w_{\sigma(j)}}\right)^{p_{j}}\right)^{\frac{1}{n !}}, 1-\prod_{\sigma \in S_{n}}\left(1-\prod_{j=1}^{n}\left(1-\left(1-\eta_{\alpha_{j}}\right)^{n w_{\sigma(j)}}\right)^{p_{j}}\right)^{\frac{1}{n !}}, \\
1-\prod_{\sigma \in S_{n}}\left(1-\prod_{j=1}^{n}\left(1-\left(1-v_{\alpha_{j}}\right)^{n w_{\sigma(j)}}\right)^{p_{j}}\right)^{\frac{1}{n !}}
\end{array}\right\}
\end{aligned}
$$

Therefore,

$$
\begin{aligned}
& \operatorname{PFWDMM}_{n w}^{P}\left(\alpha_{1}, \alpha_{2}, \cdots, \alpha_{n}\right) \\
& =\frac{1}{\sum_{j=1}^{n} p_{j}} \underset{\sigma \in S_{n}}{\otimes}\left(\bigoplus_{j=1}^{n}\left(p_{j} \alpha_{\sigma(j)}^{\left.n w_{\sigma(j)}\right)}\right)\right)^{\frac{1}{n !}} \\
& =\left\{\begin{array}{c}
1-\left(1-\prod_{\sigma \in S_{n}}\left(1-\prod_{j=1}^{n}\left(1-\left(\mu_{\alpha_{j}}\right)^{n w_{\sigma(j)}}\right)^{p_{j}}\right)^{\frac{1}{n !}}\right)^{\frac{1}{\sum_{j=1}^{n} p_{j}}}, \\
\left(1-\prod_{\sigma \in S_{n}}\left(1-\prod_{j=1}^{n}\left(1-\left(1-\eta_{\alpha_{j}}\right)^{n w_{\sigma(j)}}\right)^{p_{j}}\right)^{\frac{1}{n !}}\right)^{\frac{1}{\sum_{j=1}^{n} p_{j}}}, \\
\left(1-\prod_{\sigma \in S_{n}}\left(1-\prod_{j=1}^{n}\left(1-\left(1-v_{\alpha_{j}}\right)^{n w_{\sigma(j)}}\right)^{p_{j}}\right)^{\frac{1}{n !}}\right)^{\frac{1}{\sum_{j=1}^{n} p_{j}}}
\end{array}\right\}
\end{aligned}
$$

Hence, (35) is kept. 
Example 4. Let $(0.43,0.36,0.19),(0.79,0.02,0.10),(0.44,0.45,0.08)$ be three PFNs, and $\mathrm{P}=(0.2,0.3,0.5)$, $\sum_{j=1}^{3} p_{j}=1$ and $w=(0.4,0.3,0.3)$. Then, according to (35), we have

$$
\begin{aligned}
& \operatorname{PFWDMM}_{(0.4,0.3,0.3)}^{(0.2,0.3,0)}((0.43,0.36,0.19),(0.79,0.02,0.10),(0.44,0.45,0.08)) \\
& \left.1-\left(1-\left(\begin{array}{c}
\left(1-\left(1-0.43^{1.2}\right)^{0.2} \times\left(1-0.79^{0.9}\right)^{0.3} \times\left(1-0.44^{0.9}\right)^{0.5}\right) \\
\times\left(1-\left(1-0.43^{1.2}\right)^{0.2} \times\left(1-0.44^{0.9}\right)^{0.3} \times\left(1-0.79^{0.9}\right)^{0.5}\right) \\
\times\left(1-\left(1-0.79^{0.9}\right)^{0.2} \times\left(1-0.43^{1.2}\right)^{0.3} \times\left(1-0.44^{0.9}\right)^{0.5}\right) \\
\times\left(1-\left(1-0.79^{0.9}\right)^{0.2} \times\left(1-0.44^{0.9}\right)^{0.3} \times\left(1-0.43^{1.2}\right)^{0.5}\right) \\
\times\left(1-\left(1-0.44^{0.9}\right)^{0.2} \times\left(1-0.43^{1.2}\right)^{0.3} \times\left(1-0.79^{0.9}\right)^{0.5}\right) \\
\times\left(1-\left(1-0.44^{0.9}\right)^{0.2} \times\left(1-0.79^{0.9}\right)^{0.3} \times\left(1-0.43^{1.2}\right)^{0.5}\right)
\end{array}\right)\right)^{\frac{1}{3 !}}\right) \\
& =\left\{\left(\begin{array}{l}
\left(1-\left(1-(1-0.36)^{1.2}\right)^{0.2} \times\left(1-(1-0.02)^{0.9}\right)^{0.3} \times\left(1-(1-0.45)^{0.9}\right)^{0.5}\right) \\
\times\left(1-\left(1-(1-0.36)^{1.2}\right)^{0.2} \times\left(1-(1-0.45)^{0.9}\right)^{0.3} \times\left(1-(1-0.02)^{0.9}\right)^{0.5}\right) \\
\times\left(1-\left(1-(1-0.02)^{0.9}\right)^{0.2} \times\left(1-(1-0.36)^{1.2}\right)^{0.3} \times\left(1-(1-0.45)^{0.9}\right)^{0.5}\right) \\
\times\left(1-\left(1-(1-0.02)^{0.9}\right)^{0.2} \times\left(1-(1-0.45)^{0.9}\right)^{0.3} \times\left(1-(1-0.36)^{1.2}\right)^{0.5}\right) \\
\times\left(1-\left(1-(1-0.45)^{0.9}\right)^{0.2} \times\left(1-(1-0.36)^{1.2}\right)^{0.3} \times\left(1-(1-0.02)^{0.9}\right)^{0.5}\right) \\
\times\left(1-\left(1-(1-0.45)^{0.9}\right)^{0.2} \times\left(1-(1-0.02)^{0.9}\right)^{0.3} \times\left(1-(1-0.36)^{1.2}\right)^{0.5}\right)
\end{array}\right)^{\frac{1}{3 !}}\right)^{\frac{1}{1}} \\
& 1-\left(\begin{array}{l}
\left(1-\left(1-(1-0.19)^{1.2}\right)^{0.2} \times\left(1-(1-0.10)^{0.9}\right)^{0.3} \times\left(1-(1-0.08)^{0.9}\right)^{0.5}\right) \\
\times\left(1-\left(1-(1-0.19)^{1.2}\right)^{0.2} \times\left(1-(1-0.08)^{0.9}\right)^{0.3} \times\left(1-(1-0.10)^{0.9}\right)^{0.5}\right) \\
\times\left(1-\left(1-(1-0.10)^{0.9}\right)^{0.2} \times\left(1-(1-0.19)^{1.2}\right)^{0.3} \times\left(1-(1-0.08)^{0.9}\right)^{0.5}\right) \\
\times\left(1-\left(1-(1-0.10)^{0.9}\right)^{0.2} \times\left(1-(1-0.08)^{0.9}\right)^{0.3} \times\left(1-(1-0.19)^{1.2}\right)^{0.5}\right) \\
\times\left(1-\left(1-(1-0.08)^{0.9}\right)^{0.2} \times\left(1-(1-0.19)^{1.2}\right)^{0.3} \times\left(1-(1-0.10)^{0.9}\right)^{0.5}\right) \\
\times\left(1-\left(1-(1-0.08)^{0.9}\right)^{0.2} \times\left(1-(1-0.10)^{0.9}\right)^{0.3} \times\left(1-(1-0.19)^{1.2}\right)^{0.5}\right)
\end{array}\right) \\
& =(0.5946,0.1586,0.0560)
\end{aligned}
$$

It is clear that the PFWDMM operator satisfies the following two properties.

Theorem 14 (Boundedness). Assume that $\alpha_{j}(j=1,2, \cdots, n)$ is a group of PFNs. If

$$
\alpha^{-}=\min _{j} \alpha_{j}, \alpha^{+}=\max _{j} \alpha_{j}
$$

Then

$$
\alpha^{-} \leq \operatorname{PFDWMM}_{n w}^{P}\left(\alpha_{1}, \alpha_{2}, \cdots, \alpha_{n}\right) \leq \alpha^{+}
$$


Theorem 15 (Monotonicity). Assume that $\alpha_{j}(j=1,2, \cdots, n)$ and $\alpha^{\prime}{ }_{j}(j=1,2, \cdots, n)$ are two groups of PFNs, and let $\alpha_{j} \leq \alpha^{\prime}{ }_{j}$ for all $j$. Then,

$$
\operatorname{PFWDMM}_{n w}^{P}\left(\alpha_{1}, \alpha_{2}, \cdots, \alpha_{n}\right) \leq \operatorname{PFWDMM}_{n w}^{P}\left(\alpha^{\prime}{ }_{1}, \alpha^{\prime}{ }_{2}, \cdots, \alpha^{\prime}{ }_{n}\right) .
$$

\section{Models for MADM with PFNs}

According to the PFWMM (PFWDMM) operators, an MADM model with PFNs is briefly introduced in this part. Assume there are $m$ alternatives $A=\left\{A_{1}, A_{2}, \cdots, A_{m}\right\}$, and $n$ attributes $G=\left\{G_{1}, G_{2}, \cdots, G_{n}\right\}$ with a weighting vector $\omega=\left(\omega_{1}, \omega_{2}, \cdots, \omega_{n}\right)$, where $\omega_{j} \in[0,1], \sum_{j=1}^{n} \omega_{j}=1$. Construct the picture fuzzy decision matrix $\widetilde{R}=\left(\widetilde{r}_{i j}\right)_{m \times n}=\left(\mu_{i j}, \eta_{i j}, v_{i j}\right)_{m \times n^{\prime}}$, where $\mu_{i j}$ means the positive-membership degree function that the alternative $A_{i}$ meets the attribute $G_{j}, \eta_{i j}$ is the neutral-membership degree function that the alternative $A_{i}$ does not meet the attribute $G_{j}, v_{i j}$ denotes the negative-membership degree function that the alternative $A_{i}$ does not meet the attribute $G_{j}$, $\mu_{i j} \in[0,1], \eta_{i j} \in[0,1] v_{i j} \in[0,1], \mu_{i j}+\eta_{i j}+v_{i j} \leq 1, \pi_{i j}=1-\left(\mu_{i j}+\eta_{i j}+v_{i j}\right) i=1,2, \cdots, m$, $j=1,2, \cdots, n$.

Afterwards, we utilize the PFWMM (PFDWMM) operator to solve MADM problems with PFN information.

Step 1. We fuse the PFN information given in matrix $\widetilde{R}$ by using the PFWMM operator

$$
\begin{aligned}
& \alpha_{i}=\operatorname{PFWMM}_{n w}^{P}\left(\widetilde{r}_{i 1}, \widetilde{r}_{i 2}, \cdots, \widetilde{r}_{i n}\right) \\
& =\left(\begin{array}{l}
\left.\frac{1}{n !}\left(\bigoplus_{\sigma \in S_{n}}\left(\prod_{j=1}^{n}\left(n w_{\sigma(j)} \widetilde{r}_{i j}\right)^{p_{j}}\right)\right)\right)^{\frac{1}{\sum_{j=1}^{n} p_{j}}} \\
=\left\{\begin{array}{c}
1-\left(1-\prod_{\sigma \in S_{n}}\left(1-\prod_{j=1}^{n}\left(1-\left(1-\mu_{\alpha_{i j}}\right)^{n w_{\sigma(j)}}\right)^{p_{j}}\right)^{\frac{1}{n !}}\right)^{\frac{1}{\sum_{j=1}^{n} p_{j}}}, \\
\left.1-\left(1-\prod_{j=1}^{n}\left(1-\left(\eta_{\alpha_{i j}}\right)^{n w_{\sigma(j)}}\right)^{p_{j}}\right)^{\frac{1}{n !}}\right)^{\frac{1}{\sum_{j=1}^{n} p_{j}}}, \\
\left.\left.1-\prod_{j \in S_{n}}^{n}\left(1-\left(v_{\alpha_{i j}}\right)^{n w_{\sigma(j)}}\right)^{p_{j}}\right)^{\frac{1}{n !}}\right)^{\frac{1}{\sum_{j=1}^{n} p_{j}}}
\end{array}\right\}, i=1, \ldots, m .
\end{array}\right.
\end{aligned}
$$

Or the PFWDMM operator

$$
\begin{aligned}
& \operatorname{PFWDMM}_{n w}^{P}\left(\alpha_{1}, \alpha_{2}, \cdots, \alpha_{n}\right) \\
& =\frac{1}{\sum_{j=1}^{n} p_{j}} \underset{\sigma \in S_{n}}{\otimes}\left(\bigoplus_{j=1}^{n}\left(p_{j} \alpha_{\sigma(j)}^{\left.n w_{\sigma(j)}\right)}\right)\right)^{\frac{1}{n !}} \\
& =\left\{\begin{array}{l}
1-\left(1-\prod_{\sigma \in S_{n}}\left(1-\prod_{j=1}^{n}\left(1-\left(\mu_{\alpha_{j}}\right)^{n w_{\sigma(j)}}\right)^{p_{j}}\right)^{\frac{1}{n !}}\right)^{\frac{1}{\sum_{j=1}^{n} p_{j}}}, \\
\left(1-\prod_{\sigma \in S_{n}}\left(1-\prod_{j=1}^{n}\left(1-\left(1-\eta_{\alpha_{j}}\right)^{n w_{\sigma(j)}}\right)^{p_{j}}\right)^{\frac{1}{n !}}\right)^{\frac{1}{\Sigma_{j=1}^{n} p_{j}}}, \\
\left(1-\prod_{\sigma \in S_{n}}\left(1-\prod_{j=1}^{n}\left(1-\left(1-v_{\alpha_{j}}\right)^{n w_{\sigma(j)}}\right)^{p_{j}}\right)^{\frac{1}{n !}}\right)^{\frac{1}{\Sigma_{j=1}^{n} p_{j}}}
\end{array}\right\}, i=1,2, \cdots, m .
\end{aligned}
$$


to obtain the overall preference results $\alpha_{i}(i=1,2, \cdots, m)$ of the alternative $A_{i}$.

Step 2. Compute the score values $S\left(\alpha_{i}\right)(i=1,2, \cdots, m)$ of the overall PFNs $\alpha_{i}(i=1,2, \cdots, m)$ to order all the alternatives $A_{i}(i=1,2, \cdots, m)$. If two scores $S\left(\alpha_{i}\right)$ and $S\left(\alpha_{j}\right)$ are equal, we can compute the accuracy values $H\left(\alpha_{i}\right)$ of the overall PFNs $\alpha_{i}$, and then order the all the alternatives $A_{i}$.

Step 3. Order all the alternatives $A_{i}(i=1,2, \cdots, m)$ and select the best choice by $S\left(\alpha_{i}\right)$ $(i=1,2, \cdots, m)$.

Step 4. End.

\section{Numerical Example and Comparative Analysis}

\subsection{A Numerical Example}

As a transitional state, China has implemented reform and an opening-up policy for more than 30 years. During this period, China's economy has made marvelous achievements, and so did reform in financial circles. However, people still worry about the accumulation of financial risks and other factors that make a financial system unstable. China did successfully bear the impact of the global financial crisis in 2008; however, this does not mean that our financial system has the ability to resist any risk. In fact, there are many potential factors that can make our financial system unstable. Thus, in this section, we shall present a numerical example for evaluating financial investment risk with IVPULNs in order to illustrate the method proposed in this paper. The project's aim is to evaluate the best financial investment alternatives from the different financial investment alternatives in an enterprise financial risk environment. In order to select most desirable enterprise, the desirability levels of five possible financial investment alternatives $A_{i}(i=1,2,3,4,5)$ are evaluated. The team of experts must make a decision according to the following four attributes: (1) $\mathrm{G}_{1}$ is the market risk; (2) $G_{2}$ is the enterprise's operation and management risk; (3) $G_{3}$ is the enterprise's assets structure risk; and (4) $\mathrm{G}_{4}$ is the environmental risk. The experts use the above attributes to evaluate the five possible financial investment alternatives $A_{i}(i=1,2, \cdots, 5)$ by using the PFNs by the decision-makers under the above four attributes (whose weighting vector is $\omega=(0.3,0.2,0.4,0.1)$ ), and construct the following matrix $\widetilde{R}=\left(\widetilde{r}_{i j}\right)_{5 \times 4}$ as shown in Table 1 .

Table 1. The picture fuzzy number (PFN) information decision matrix.

\begin{tabular}{cccccc}
\hline & $\mathbf{A}_{\mathbf{1}}$ & $\mathbf{A}_{\mathbf{2}}$ & $\mathbf{A}_{\mathbf{3}}$ & $\mathbf{A}_{\mathbf{4}}$ & $\mathbf{A}_{\mathbf{5}}$ \\
\hline $\mathrm{G}_{1}$ & $(0.43,0.36,0.19)$ & $(0.43,0.32,0.18)$ & $(0.71,0.23,0.01)$ & $(0.25,0.49,0.15)$ & $(0.50,0.45,0.03)$ \\
$\mathrm{G}_{2}$ & $(0.79,0.02,0.01)$ & $(0.73,0.04,0.11)$ & $(0.87,0.02,0.03)$ & $(0.64,0.12,0.13)$ & $(0.78,0.03,0.11)$ \\
$\mathrm{G}_{3}$ & $(0.43,0.45,0.08)$ & $(0.03,0.62,0.33)$ & $(0.04,0.55,0.30)$ & $(0.01,0.69,0.25)$ & $(0.03,0.57,0.26)$ \\
$\mathrm{G}_{4}$ & $(0.18,0.39,0.04)$ & $(0.53,0.25,0.18)$ & $(0.48,0.26,0.16)$ & $(0.02,0.54,0.26)$ & $(0.13,0.65,0.19)$ \\
\hline
\end{tabular}

To select the most desirable financial investment alternative, we use the PFWMM (PFWDMM) operator to solve the MADM model with PFNs. The computing steps are listed as follows.

- $\quad$ Step 1. Based on Table 1 , fuse all PFNs $\tilde{r}_{i j}(j=1,2, \cdots, n)$ by utilizing the PFWMM (PFWDMM) operator to obtain the overall PFNs $\alpha_{i}(i=1,2,3,4,5)$ of the financial investment alternative $A_{i}$. The fused values are listed in Table 2.

- Step 2. Based on the fused values shown in Table 2, the score values of the financial investment alternatives are given in Table 3.

- Step 3. Based on the score values of the overall alternatives (Table 4), we can rank all the alternatives, and the ranking of the financial investment alternatives is slightly different. 
Table 2. The fused values of the financial investment alternatives by the picture fuzzy weighted Muirhead mean (PFWMM) operator and the picture fuzzy weighted dual Muirhead mean (PFWDMM) operator.

\begin{tabular}{ccc}
\hline & PFWMM & PFWDMM \\
\hline $\mathrm{A}_{1}$ & $(0.4056,0.3604,0.1213)$ & $(0.5234,0.2365,0.0555)$ \\
$\mathrm{A}_{2}$ & $(0.3036,0.3700,0.2605)$ & $(0.5571,0.2222,0.1703)$ \\
$\mathrm{A}_{3}$ & $(0.3719,0.3239,0.1844)$ & $(0.6637,0.1880,0.0666)$ \\
$\mathrm{A}_{4}$ & $(0.0965,0.5274,0.2694)$ & $(0.2934,0.3709,0.1701)$ \\
$\mathrm{A}_{5}$ & $(0.2166,0.4819,0.2210)$ & $(0.4738,0.3379,0.1087)$ \\
\hline
\end{tabular}

Table 3. The score functions of the financial investment alternatives.

\begin{tabular}{ccc}
\hline & PFWMM & PFWDMM \\
\hline $\mathrm{A}_{1}$ & 0.2843 & 0.4679 \\
$\mathrm{~A}_{2}$ & 0.0431 & 0.3867 \\
$\mathrm{~A}_{3}$ & 0.1874 & 0.5971 \\
$\mathrm{~A}_{4}$ & -0.1730 & 0.1233 \\
$\mathrm{~A}_{5}$ & -0.0044 & 0.3651 \\
\hline
\end{tabular}

Table 4. Ordering of the financial investment alternatives.

\begin{tabular}{cc}
\hline & Ordering \\
\hline PFWMM & $A_{1}>A_{3}>A_{2}>A_{5}>A_{4}$ \\
PFWDMM & $A_{3}>A_{1}>A_{2}>A_{5}>A_{4}$ \\
\hline
\end{tabular}

\subsection{Comparative Analysis}

In addition, a comparative analysis was made between the PFWMM(PFWDMM) operator and the PFWA and PFWG operators defined by Wei [31]. The comparative results are given in Table 5.

Table 5. Ranking of the financial investment alternatives.

\begin{tabular}{cc}
\hline & Ordering \\
\hline PFWA & $A_{3}>A_{1}>A_{5}>A_{2}>A_{4}$ \\
PFWG & $A_{1}>A_{3}>A_{5}>A_{2}>A_{4}$ \\
\hline
\end{tabular}

From above, we can see that the fused values are slightly different in the ordering of the alternatives to show the accuracy and scientific merit of the proposed approaches. However, the PFWA and PFWG operators have the limitation of not considering the relationships between the attributes in the fused information. Our defined PFWMM and PFWDMM operators have the advantage of taking the interaction relationships among any number of attributes into account, and can be more effective and accurate.

\section{Conclusions}

Aggregation operators have become a hot issue and an important tool in the decision-making fields in recent years. However, they still have some limitations in practical applications. For example, some aggregation operators suppose that the attributes are independent of each other. However, the MM operator and the dual MM operator have a prominent characteristic: they can consider the interaction relationships among any number of attributes by a parameter vector $\lambda$. According to the MM operator and the dual MM operator, in this article, we defined some new MM and DMM aggregation operators to deal with MADM problems under a PFN environment, including the PFMM operator, the PFWMM operator, the PFDMM operator and the PFWDMM operator. Of course, the precious merits of these defined operators are investigated. Moreover, we have adopted 
PFWMM and PFWDMM operators to build some decision-making models to handle picture fuzzy MADM problems. In the end, we take a concrete instance of appraising a financial investment risk to demonstrate our defined model and to verify its accuracy and scientific merit. In the future, we can apply our defined PFN aggregation operators into other decision-making fields, such as the decision-making, risk analysis, and other fields that operate in an uncertain environment [54,69-80].

Author Contributions: R.W., J.W., H.G. and G.W. conceived and worked together to achieve this work; J.W. compiled the computing program by Excel and analyzed the data; J.W. and G.W. wrote the paper; Finally, all the authors have read and approved the final manuscript.

Funding: The work was supported by the National Natural Science Foundation of China under Grant No. 71571128 and the Humanities and Social Sciences Foundation of Ministry of Education of the People's Republic of China (16XJA630005) and the Construction Plan of Scientific Research Innovation Team for Colleges and Universities in Sichuan Province (15TD0004).

Conflicts of Interest: The authors declare no conflict of interest.

\section{References}

1. Atanassov, K.T. Intuitionistic fuzzy sets. Fuzzy Sets Syst. 1986, 20, 87-96. [CrossRef]

2. Zadeh, L.A. Fuzzy Sets. Inf. Control 1965, 8, 338. [CrossRef]

3. Atanassov, K.; Gargov, G. Interval valued intuitionistic fuzzy-sets. Fuzzy Sets Syst. 1989, 31, $343-349$. [CrossRef]

4. Atanassov, K.T. Operators over interval-valued intuitionistic fuzzy sets. Fuzzy Sets Syst. 1994, 64, 159-174. [CrossRef]

5. Zhang, X.H.; Mao, X.Y.; Smarandache, F.; Park, C. On Homomorphism Theorem for Perfect Neutrosophic Extended Triplet Groups. Information 2018, 9, 237. [CrossRef]

6. Sahin, R. Normal neutrosophic multiple attribute decision making based on generalized prioritized aggregation operators. Neural Comput. Appl. 2018, 30, 3095-3115. [CrossRef]

7. Li, Z.X.; Gao, H.; Wei, G.W. Methods for Multiple Attribute Group Decision Making Based on Intuitionistic Fuzzy Dombi Hamy Mean Operators. Symmetry 2018, 10, 574. [CrossRef]

8. Wei, G.W.; Wei, C.; Wang, J.; Gao, H.; Wei, Y. Some q-rung orthopair fuzzy maclaurin symmetric mean operators and their applications to potential evaluation of emerging technology commercialization. Int. J. Intell. Syst. 2019, 34, 50-81. [CrossRef]

9. Wu, L.; Wei, G.; Gao, H.; Wei, Y. Some Interval-Valued Intuitionistic Fuzzy Dombi Hamy Mean Operators and Their Application for Evaluating the Elderly Tourism Service Quality in Tourism Destination. Mathematics 2018, 6, 294. [CrossRef]

10. Wei, G.W.; Garg, H.; Gao, H.; Wei, C. Interval-Valued Pythagorean Fuzzy Maclaurin Symmetric Mean Operators in Multiple Attribute Decision Making. IEEE Access 2018, 6, 67866-67884. [CrossRef]

11. Wei, G.W.; Wei, C.; Gao, H. Multiple Attribute Decision Making with Interval-Valued Bipolar Fuzzy Information and Their Application to Emerging Technology Commercialization Evaluation. IEEE Access 2018, 6, 60930-60955. [CrossRef]

12. Wei, G.W.; Gao, H. The Generalized Dice Similarity Measures for Picture Fuzzy Sets and Their Applications. Informatica 2018, 29, 107-124. [CrossRef]

13. Garg, H.; Nancy. Non-linear programming method for multi-criteria decision making problems under interval neutrosophic set environment. Appl. Intell. 2018, 48, 2199-2213. [CrossRef]

14. Wang, J.; Wei, G.W.; Wei, Y. Models for Green Supplier Selection with Some 2-Tuple Linguistic Neutrosophic Number Bonferroni Mean Operators. Symmetry 2018, 10, 131. [CrossRef]

15. Ullah, K.; Mahmood, T.; Jan, N. Similarity Measures for T-Spherical Fuzzy Sets with Applications in Pattern Recognition. Symmetry 2018, 10, 193. [CrossRef]

16. Liu, P.D.; You, X.L. Some linguistic neutrosophic Hamy mean operators and their application to multi-attribute group decision making. PLoS ONE 2018, 13, e0193027. [CrossRef] [PubMed]

17. Cuong, B.C.; Kreinovich, V. Picture Fuzzy Sets-A new concept for computational intelligence problems. In Proceedings of the 2013 Third World Congress on Information and Communication Technologies (WICT 2013), Hanoi, Vietnam, 15-18 December 2013; pp. 1-6.

18. Singh, P. Correlation coefficients for picture fuzzy sets. J. Intell. Fuzzy Syst. 2015, 28, 591-604. 
19. Son, L.H. DPFCM: A novel distributed picture fuzzy clustering method on picture fuzzy sets. Expert Syst. Appl. 2015, 42, 51-66. [CrossRef]

20. Son, L.H. Generalized picture distance measure and applications to picture fuzzy clustering. Appl. Soft Comput. 2016, 46, 284-295. [CrossRef]

21. Thong, N.T.; Son, L.H. HIFCF: An effective hybrid model between picture fuzzy clustering and intuitionistic fuzzy recommender systems for medical diagnosis. Expert Syst. Appl. 2015, 42, 3682-3701. [CrossRef]

22. Wei, G.W. Picture fuzzy cross-entropy for multiple attribute decision making problems. J. Bus. Econ. Manag. 2016, 17, 491-502. [CrossRef]

23. Thong, P.H.; Son, L.H. A novel automatic picture fuzzy clustering method based on particle swarm optimization and picture composite cardinality. Knowl.-Based Syst. 2016, 109, 48-60. [CrossRef]

24. Wei, G.W. Some Cosine Similarity Measures for Picture Fuzzy Sets and Their Applications to Strategic Decision Making. Informatica 2017, 28, 547-564. [CrossRef]

25. Wei, G.W.; Lin, R.; Wang, H.J. Distance and similarity measures for hesitant interval-valued fuzzy sets. J. Intell. Fuzzy Syst. 2014, 27, 19-36.

26. Wei, G.W.; Wei, Y. Similarity measures of Pythagorean fuzzy sets based on the cosine function and their applications. Int. J. Intell. Syst. 2018, 33, 634-652. [CrossRef]

27. Wu, H.B.; Yuan, Y.; Wei, L.J.; Pei, L.D. On entropy, similarity measure and cross-entropy of single-valued neutrosophic sets and their application in multi-attribute decision making. Soft Comput. 2018, 22, 7367-7376. [CrossRef]

28. Ye, J. Multiple-attribute decision-making method using similarity measures of single-valued neutrosophic hesitant fuzzy sets based on least common multiple cardinality. J. Intell. Fuzzy Syst. 2018, 34, 4203-4211. [CrossRef]

29. Zhai, Y.L.; Xu, Z.S.; Liao, H.C. Measures of Probabilistic Interval-Valued Intuitionistic Hesitant Fuzzy Sets and the Application in Reducing Excessive Medical Examinations. IEEE Trans. Fuzzy Syst. 2018, 26, 1651-1670. [CrossRef]

30. Wei, G.W. Some similarity measures for picture fuzzy sets and their applications. Iran. J. Fuzzy Syst. 2018, 15, 77-89.

31. Wei, G.W. Picture fuzzy aggregation operators and their application to multiple attribute decision making. J. Intell. Fuzzy Syst. 2017, 33, 713-724. [CrossRef]

32. Wei, G.W.; Alsaadi, F.E.; Hayat, T.; Alsaedi, A. Bipolar Fuzzy Hamacher Aggregation Operators in Multiple Attribute Decision Making. Int. J. Fuzzy Syst. 2018, 20, 1-12. [CrossRef]

33. Wei, G.W.; Gao, H.; Wang, J.; Huang, Y.H. Research on Risk Evaluation of Enterprise Human Capital Investment with Interval-Valued Bipolar 2-Tuple Linguistic Information. IEEE Access 2018, 6, 35697-35712. [CrossRef]

34. Wei, G.W.; Lu, M. Pythagorean Fuzzy Maclaurin Symmetric Mean Operators in Multiple Attribute Decision Making. Int. J. Intell. Syst. 2018, 33, 1043-1070. [CrossRef]

35. Wei, G.W.; Lu, M. Pythagorean fuzzy power aggregation operators in multiple attribute decision making. Int. J. Intell. Syst. 2018, 33, 169-186. [CrossRef]

36. Wei, G.W.; Lu, M.; Tang, X.Y.; Wei, Y. Pythagorean hesitant fuzzy Hamacher aggregation operators and their application to multiple attribute decision making. Int. J. Intell. Syst. 2018, 33, 1197-1233. [CrossRef]

37. Wu, S.J.; Wang, J.; Wei, G.W.; Wei, Y. Research on Construction Engineering Project Risk Assessment with Some 2-Tuple Linguistic Neutrosophic Hamy Mean Operators. Sustainability 2018, 10, 1536. [CrossRef]

38. Wei, G.W. Picture Fuzzy Hamacher Aggregation Operators and their Application to Multiple Attribute Decision Making. Fundam. Inform. 2018, 157, 271-320. [CrossRef]

39. Gao, H.; Wei, G.W.; Huang, Y.H. Dual Hesitant Bipolar Fuzzy Hamacher Prioritized Aggregation Operators in Multiple Attribute Decision Making. IEEE Access 2018, 6, 11508-11522. [CrossRef]

40. Wu, S.J.; Wei, G.W. Pythagorean fuzzy Hamacher aggregation operators and their application to multiple attribute decision making. Int. J. Knowl.-Based Intell. Eng. Syst. 2017, 21, 189-201. [CrossRef]

41. Lu, M.; Wei, G.W.; Alsaadi, F.E.; Hayat, T.; Alsaedi, A. Hesitant pythagorean fuzzy hamacher aggregation operators and their application to multiple attribute decision making. J. Intell. Fuzzy Syst. 2017, 33, 1105-1117. [CrossRef]

42. Wei, G.W.; Lu, M. Dual hesitant pythagorean fuzzy Hamacher aggregation operators in multiple attribute decision making. Arch. Control Sci. 2017, 27, 365-395. [CrossRef] 
43. Zhang, X.Y.; Wang, X.K.; Yu, S.M.; Wang, J.Q.; Wang, T.L. Location selection of offshore wind power station by consensus decision framework using picture fuzzy modelling. J. Clean. Prod. 2018, 202, 980-992. [CrossRef]

44. Wei, G.W. TODIM Method for Picture Fuzzy Multiple Attribute Decision Making. Informatica 2018, 29, 555-566. [CrossRef]

45. Wang, L.; Peng, J.J.; Wang, J.Q. A multi-criteria decision-making framework for risk ranking of energy performance contracting project under picture fuzzy environment. J. Clean. Prod. 2018, 191, 105-118. [CrossRef]

46. Wei, G.W.; Alsaadi, F.E.; Hayat, T.; Alsaedi, A. Projection models for multiple attribute decision making with picture fuzzy information. Int. J. Mach. Learn. Cybern. 2018, 9, 713-719. [CrossRef]

47. Wei, G.W. Picture 2-Tuple Linguistic Bonferroni Mean Operators and Their Application to Multiple Attribute Decision Making. Int. J. Fuzzy Syst. 2017, 19, 997-1010. [CrossRef]

48. Wei, G.W.; Alsaadi, F.E.; Hayat, T.; Alsaedi, A. Picture 2-tuple linguistic aggregation operators in multiple attribute decision making. Soft Comput. 2018, 22, 989-1002. [CrossRef]

49. Wei, G.W. Picture uncertain linguistic Bonferroni mean operators and their application to multiple attribute decision making. Kybernetes 2017, 46, 1777-1800. [CrossRef]

50. Wang, R.; Li, Y.L. Picture Hesitant Fuzzy Set and Its Application to Multiple Criteria Decision-Making. Symmetry 2018, 10, 295. [CrossRef]

51. Torra, V. Hesitant Fuzzy Sets. Int. J. Intell. Syst. 2010, 25, 529-539. [CrossRef]

52. Wang, Z.X.; Li, J. Correlation Coefficients of Probabilistic Hesitant Fuzzy Elements and Their Applications to Evaluation of the Alternatives. Symmetry 2017, 9, 259. [CrossRef]

53. Wei, G.W. Interval-valued dual hesitant fuzzy uncertain linguistic aggregation operators in multiple attribute decision making. J. Intell. Fuzzy Syst. 2017, 33, 1881-1893. [CrossRef]

54. Wei, G.W.; Alsaadi, F.E.; Hayat, T.; Alsaedi, A. Hesitant bipolar fuzzy aggregation operators in multiple attribute decision making. J. Intell. Fuzzy Syst. 2017, 33, 1119-1128. [CrossRef]

55. Wu, S.M.; Liu, H.C.; Wang, L.E. Hesitant fuzzy integrated MCDM approach for quality function deployment: A case study in electric vehicle. Int. J. Prod. Res. 2017, 55, 4436-4449. [CrossRef]

56. Gitinavard, H.; Ghaderi, H.; Pishvaee, M.S. Green supplier evaluation in manufacturing systems: A novel interval-valued hesitant fuzzy group outranking approach. Soft Comput. 2018, 22, 6441-6460. [CrossRef]

57. Muirhead, R.F. Some methods applicable to identities and inequalities of symmetric algebraic functions of $\mathrm{n}$ letters. Proc. Edinb. Math. Soc. 1902, 21, 144-162. [CrossRef]

58. Bonferroni, C. Sulle medie multiple di potenze. Boll. Mat. Ital. 1950, 5, 267-270.

59. Wei, G.W.; Zhao, X.F.; Lin, R.; Wang, H.J. Uncertain linguistic Bonferroni mean operators and their application to multiple attribute decision making. Appl. Math. Model. 2013, 37, 5277-5285. [CrossRef]

60. Jiang, X.P.; Wei, G.W. Some Bonferroni mean operators with 2-tuple linguistic information and their application to multiple attribute decision making. J. Intell. Fuzzy Syst. 2014, 27, 2153-2162.

61. Deng, X.M.; Wei, G.W.; Gao, H.; Wang, J. Models for Safety Assessment of Construction Project with Some 2-Tuple Linguistic Pythagorean Fuzzy Bonferroni Mean Operators. IEEE Access 2018, 6, 52105-52137. [CrossRef]

62. Tang, X.Y.; Wei, G.W. Models for Green Supplier Selection in Green Supply Chain Management with Pythagorean 2-Tuple Linguistic Information. IEEE Access 2018, 6, 18042-18060. [CrossRef]

63. Wang, J.; Tang, X.Y.; Wei, G.W. Models for Multiple Attribute Decision-Making with Dual Generalized Single-Valued Neutrosophic Bonferroni Mean Operators. Algorithms 2018, 11, 2. [CrossRef]

64. Wang, J.; Wei, G.W.; Gao, H. Approaches to Multiple Attribute Decision Making with Interval-Valued 2-Tuple Linguistic Pythagorean Fuzzy Information. Mathematics 2018, 6, 201. [CrossRef]

65. Cuong, B.C.; Hai, P.V. Some fuzzy logic operators for picture fuzzy sets. In Proceedings of the 2015 Seventh International Conference on Knowledge and Systems Engineering (KSE), Ho Chi Minh City, Vietnam, 8-10 October 2015; pp. 132-137.

66. Xu, Z.S.; Yager, R.R. Some geometric aggregation operators based on intuitionistic fuzzy sets. Int. J. Gen. Syst. 2006, 35, 417-433. [CrossRef]

67. Xu, Z.S. Intuitionistic fuzzy aggregation operators. IEEE Trans. Fuzzy Syst. 2007, 15, 1179-1187.

68. Qin, J.D.; Liu, X.W. 2-tuple linguistic Muirhead mean operators for multiple attribute group decision making and its application to supplier selection. Kybernetes 2016, 45, 2-29. [CrossRef] 
69. Ye, J. Simplified neutrosophic harmonic averaging projection-based method for multiple attribute decision-making problems. Int. J. Mach. Learn. Cybern. 2017, 8, 981-987. [CrossRef]

70. Wei, G.W. Pythagorean fuzzy interaction aggregation operators and their application to multiple attribute decision making. J. Intell. Fuzzy Syst. 2017, 33, 2119-2132. [CrossRef]

71. Ye, J. Bidirectional projection method for multiple attribute group decision making with neutrosophic numbers. Neural Comput. Appl. 2017, 28, 1021-1029. [CrossRef]

72. Wei, G.W.; Lu, M.; Alsaadi, F.E.; Hayat, T.; Alsaedi, A. Pythagorean 2-tuple linguistic aggregation operators in multiple attribute decision making. J. Intell. Fuzzy Syst. 2017, 33, 1129-1142. [CrossRef]

73. Ye, J. Some Weighted Aggregation Operators of Trapezoidal Neutrosophic Numbers and Their Multiple Attribute Decision Making Method. Informatica 2017, 28, 387-402. [CrossRef]

74. Wei, G.W.; Alsaadi, F.E.; Hayat, T.; Alsaedi, A. A Linear Assignment Method for Multiple Criteria Decision Analysis with Hesitant Fuzzy Sets Based on Fuzzy Measure. Int. J. Fuzzy Syst. 2017, 19, 607-614. [CrossRef]

75. Wei, G.W.; Wang, J.M. A comparative study of robust efficiency analysis and Data Envelopment Analysis with imprecise data. Expert Syst. Appl. 2017, 81, 28-38. [CrossRef]

76. Jankowski, J.; Kazienko, P.; Watrobski, J.; Lewandowska, A.; Ziemba, P.; Ziolo, M. Fuzzy multi-objective modeling of effectiveness and user experience in online advertising. Expert Syst. Appl. 2016, 65, 315-331. [CrossRef]

77. Deng, X.M.; Wang, J.; Wei, G.W.; Lu, M. Models for Multiple Attribute Decision Making with Some 2-Tuple Linguistic Pythagorean Fuzzy Hamy Mean Operators. Mathematics 2018, 6, 236. [CrossRef]

78. Medina, J.; Ojeda-Aciego, M. Multi-adjoint t-concept lattices. Inf. Sci. 2010, 180, 712-725. [CrossRef]

79. Pozna, C.; Minculete, N.; Precup, R.E.; Koczy, L.T.; Ballagi, A. Signatures: Definitions, operators and applications to fuzzy modelling. Fuzzy Sets Syst. 2012, 201, 86-104. [CrossRef]

80. Kumar, A.; Kumar, D.; Jarial, S.K. A hybrid clustering method based on improved artificial bee colony and fuzzy C-means algorithm. Int. J. Artif. Intell. 2017, 15, 40-60.

(C) 2018 by the authors. Licensee MDPI, Basel, Switzerland. This article is an open access article distributed under the terms and conditions of the Creative Commons Attribution (CC BY) license (http:// creativecommons.org/licenses/by/4.0/). 